GRÖ PRICE

$\boldsymbol{s}$

$X-611-65-249$

CFSTI PRICE(S) \$

Hard copy $(H C) \frac{1,00}{150}$

IASA TMX-5\%231

\begin{abstract}
THE LOW ENERGY SPECTRUM
OF COSMIC RAYS AS AN INDICATOR

OF PRIMARY SOURCE CHARACTERISTICS

AND INTERSTELLAR PROPAGATION

ff 653 Juty 65
\end{abstract}

BY

V. K. BALASUBRAHMANYAN

E. BOLDT

R. A. R. PALMEIRA

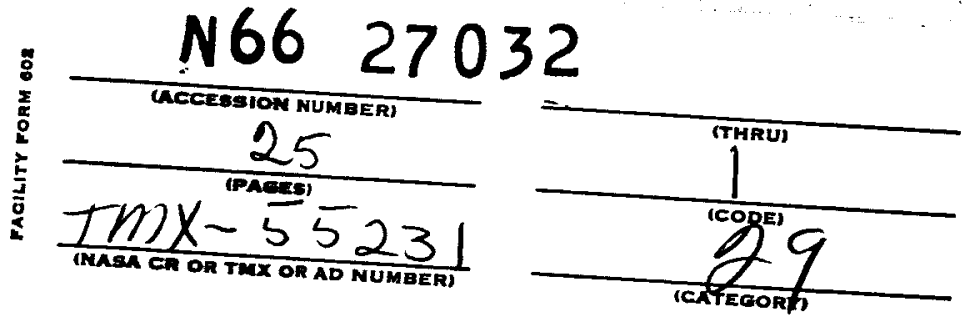

IUNE 21, 1965

$N A S A$

GODDARD SPACE FLIGHT CENTER

GREENBELT, MD.

To be submitted to The Physical Review

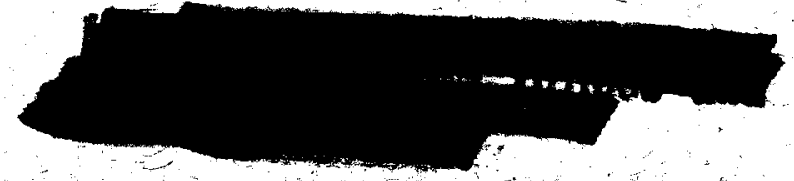




\section{THE LOW ENERGY SPECTRUM OF COSMIC RAYS AS AN} INDICATOR OF PRIMARY SOURCE CHARACTERISTICS AND INTERSTELLAR PROPAGATION

V. K. Balasubrahmanyan, E. Boldt, and R. A. R. Palmeira NASA - Goddard Space Flight Center Greenbelt, Maryland

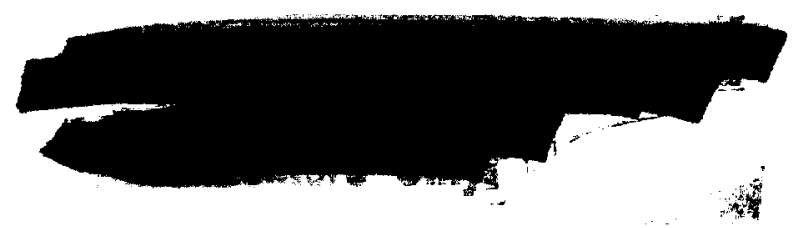




\title{
THE LOW ENERGY SPECTRUM OF COSMIC RAYS AS AN INDICATOR OF PRIMARY SOURCE CHARACTERISTICS AND INTERSTELLAR PROPAGATION
}

\author{
by \\ V. K. Balasubrahmanyan, E. Boldt, \\ and R. A. R. Palmeira \\ NASA - Goddard Space Flight Center \\ Greenbelt, Maryland
}

\section{ABSTRACT}

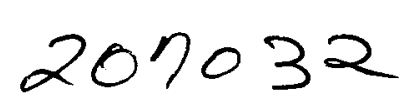

The low energy spectra of protons and helium nuclei, recently measured by McDonald and Ludwig, and by Fan, Gloeckler and Simpson, are corrected herein for solar modulation and diffusive passage through interstellar matter. The resulting source spectrum is exhibited and compared with the spectrum of accelerated particles inferred from high energy data. This analysis yields a transmission function for the source environment which is similar in form to the velocity dependent solar modulation of low energy particles. 


\title{
THE LOW ENERGY SPECTRUM OF COSMIC RAYS AS AN
}

\section{INDICATOR OF PRIMARY SOURCE CHARACTERISTICS}

AND INTERSTELLAR PROPAGATION

by

\author{
V. K. Balasubrahmanyan, ${ }^{*}$ E. Boldt, \\ and
}

R. A. R. Palmeira

NASA - Goddard Space Flight Center

Greenbelt, Maryland

\section{INTRODUCTION}

In the study of galactic cosmic radiation the evolution of the observed particles can be considered in four phases ${ }^{1}$ :

(a) injection from source regions

(b) possible partial confinement in source regions

(c) motion in the interstellar medium

(d) solar modulation.

Interstellar propagation has been studied extensively by many workers $2,3,4$ from a detailed determination of the charge spectrum of the cosmic radiation. In particular, it has been found that $L_{i}, B e, B$ (called $L$ nuclei) occur in primary

* On leave from Tata Institute of Fundamental Research, India

†NAS-NASA Postdoctoral Research Associate

†NAS-NASA Postdoctoral Research Associate on leave from Centro Brasileiro de Pesquisas Fisicas, Rio de Janeiro, Brazil 
cosmic radiation with very large abundance when compared to their universal abundance. As these nuclei are easily destroyed in nuclear interactions at stellar temperatures, it is reasonable to assume that they are probably not present in the source regions, but are produced in nuclear collisions of heavier nuclei with the protons of the interstellar medium. Using the abundance of these light nuclei relative to heavier nuclei, an estimate of the mean amount of matter traversed in the interstellar medium ${ }^{5}$ has been made. For relativistic energies, a recent estimate ${ }^{6}$ which takes into account effects due to decay of unstable isotopes formed in collisions and other details of nuclear collisions, gives a value of $2.5 \mathrm{gm} / \mathrm{cm}^{2}$ as the mean amount of matter traversed by cosmic rays in the interstellar medium and is considered to be a reliable measure of this quantity. ${ }^{7}$ At low energies, however, the picture is not so clear and some experiments 8,9 seem to indicate an increase in the ratio of the abundance of $L$ nuclei to that of heavier nuclei. Kaplon and Skadron ${ }^{1}$ have interpreted the increase of this ratio as evidence that the amount of matter traversed by low energy nuclei is enhanced by a preferential confinement for low energy particles inside source regions that are surrounded by partially reflecting boundaries.

The analysis presented herein is based primarily upon recent experiments, conducted on board the IMP-I satellite, which yielded the first clear measurement of the low energy spectrum of cosmic rays. The proton spectrum thus obtained by McDonald and Ludwig ${ }^{10}$ and the helium nucleus spectrum obtained by Fan, Gloeckler and Simpson ${ }^{11}$ are found to exhibit pertinent information about source transmission and interstellar propagation of low energy particles. Since these particles lie in the interval 20-100 MeV per nucleon, ionization los is the dominant feature of their propagation in the interstellar medium, and their 
energy is thereby greatly modified. This degradation of energy is a direct sensitive indication of the quantity of matter traversed by cosmic rays. Owing to their low magnetic rigidity, these particles also serve as a probe of the magnetic structure that is characteristic of the source environment.

In this paper we define the source spectrum in an operational way. We first take the experimentally observed energy spectrum and "demodulate" it for solar cycle effects. This "demodulated" spectrum which represents the cosmic ray energy spectrum outside the solar system, is traced back through the interstellar medium by taking into account the energy loss due to ionization. The path length dispersion arising from the diffusion of cosmic rays in the interstellar medium is considered in the analysis; this involves a specification of the distribution in path lengths about the mean value of $2.5 \mathrm{gm} / \mathrm{cm}^{2}$. Finally, by comparing the source spectrum obtained in this way with the spectrum of accelerated particles inferred from high energy data, we are able to reconstruct the transmission function for the physical source environment.

\section{THE LOW ENERGY PROTON AND HELIUM}

\section{NUCLEUS SPECTRUM}

Figure 1 exhibits the proton and helium nucleus rigidity spectra obtained from recent balloon and IMP-I satellite measurements. The helium nucleus data have been normalized to the proton data using a scale factor of 7 . Although the proton and helium nucleus rigidity spectra are comparable at high rigidities, it is clear that at rigidities below about $1500 \mathrm{MV}$ the two spectra are definitely distinct. This shows that rigidity can not be used as a universal parameter for characterizing the spectrum of cosmic ray particles, at low energies. 
Figure 2 shows the proton and helium nucleus spectra in a representation based upon the kinetic energy per nucleon. In this instance, the helium nucleus data have been scaled by 5.7 to achieve a normalization to the proton data. As indicated in Figure 2, a single curve describes all the pertinent proton and helium nucleus data points. This suggests that the kinetic energy per nucleon, or any other purely velocity dependent function, might be used to completely characterize the cosmic ray spectrum at low energies. The analytical expression for the indicated curve is

$$
\frac{\mathrm{dJ}}{\mathrm{dE}}=10^{8} \mathrm{E}^{1.5}(\mathrm{E}+500)^{-4}
$$

where $\mathrm{dJ} / \mathrm{dE}$ has the units of particles $/ \mathrm{m}^{2}-$ sec-ster-MeV, and $\mathrm{E}$ is the kinetic energy per nucleon in MeV. This analytical representation of the observed spectrum is shown again as curve $A$ in Figure 3. It was constructed to meet an asymptotic constraint imposed by the very high energy data ${ }^{12}$ (i.e. E > $10^{10} \mathrm{eV}$ ); viz,

$$
\frac{\mathrm{dJ}}{\mathrm{dE}} \propto \mathrm{E}^{-2.5}
$$

The ratio of the proton flux to the helium nucleus flux at high energies is not as yet established with a precision comparable to that ascribed to this ratio for the low energies considered here. However, a comparison of the best estimates 13,14 of the cosmic ray flux at kinetic energies of $10^{11}$ to $10^{13} \mathrm{eV}$ per nucleon with helium nucleon flux measurements 15,16 indicates that the ratio at very high energies is consistent with the value used in this paper for energies 
in the interval $20 \mathrm{MeV}$ per nucleon to $1 \mathrm{BeV}$ per nucleon. In the region 2 to 15 $\mathrm{BeV}$ per nucleon, integral intensity measurements give some evidence $\mathrm{e}^{17}$ that the proton to alpha ratio might be different at these intermediate energies, but this region is not crucial to the present analysis.

For protons and helium nuclei in the low energy range the important effects to be taken into account are ionization loss in the interstellar medium and solar modulation. At low energy these processes affect the protons and helium nuclei in an identical way as regards changes per nucleon. The reasons for this are that: (i) for equal $\mathbf{Z}^{2} / \mathbf{A}$, which is the case for protons and helium nuclei, the ionization loss per nucleon is a universal function of velocity, and (ii) the low energy solar modulation is a universal function of velocity.

McDonald and Ludwig ${ }^{10}$ have deduced the galactic proton spectrum expected at Earth under the assumption of:

(i) an energy spectrum at injection of the form

$$
\mu(\epsilon)=\frac{4}{3} \pi \times 10^{-4} / \beta(1+\epsilon)^{2.5} \text { protons } / \mathrm{m}^{3}-\mathrm{BeV},
$$

where $\mu(\epsilon)$ is the density of cosmic rays of kinetic energy $\epsilon$ in $\mathrm{BeV}$ and $\beta$ is the proton velocity in units of the velocity of light;

(ii) $2.5 \mathrm{gm} / \mathrm{cm}^{2}$ of hydrogen traversed in the interstellar medium; and

(iii) a solar modulation given by

$$
\left(\frac{d J}{d \epsilon}\right)=\left(\frac{d J}{d \epsilon}\right)_{0} \exp (-0.8 / \beta)
$$


where $(\mathrm{dJ} / \mathrm{d} \epsilon)$ and $(\mathrm{dJ} / \mathrm{d} \epsilon)_{0}$ are the modulated and unmodulated spectra, respectively.

The results of these calculations by McDonald and Ludwig are shown by the dotted curve of Figure 1. By comparing their proton data with this dotted curve, they conclude that either the source spectrum is much steeper than what has been assumed or the solar modulation is weaker at the low energies experimentally observed. Using the same solar modulation, the present analysis indicates that the source spectrum at low energies is strongly affected by the transmission characteristics of the source environment.

\section{SOLAR MODULATION}

Parker ${ }^{18}$ has described the 11 -year solar modulation of cosmic rays in terms of a quasi-stationary solution to a Smoluchowski generalized diffusion equation $^{19}$ for the process of a charged particle diffusing among the magnetic kinks convected away from the sun by the plasma wind. For energies sufficiently low such that the Larmor radius $(R)$ is small compared with the linear dimension $(\ell)$ of the magnetic kinks, the solution may be expressed as

$$
\mu_{\text {in }}=\left(\mu_{\text {out }}\right) \exp (-K / \beta)
$$

where $\mu_{\text {in }}$ is the cosmic ray density at the orbit of Earth, $\mu_{\text {out }}$ is the cosmic ray density outside the solar environment, and $\mathrm{K}$ is a solar modulation parameter that is proportional to the solar wind velocity and the thickness of the solar cavity, measured in units of kink intervals.

For high enough energies such that $R>\ell$ the solution takes on the form

$$
\mu_{\text {in }}=\left(\mu_{\text {out }}\right) \exp \left\{-\left(\frac{\mathbf{K}}{\beta^{3}}\right)\left(1-\beta^{2}\right)\left(\frac{\ell Z}{R_{0} A}\right)^{2}\right\}
$$


where $R_{0} \equiv \mathrm{Mc}^{2} / \mathrm{eB}, \mathbf{M}$ is the proton mass, $B$ the magnetic field, and $\mathbf{Z}$ and $\mathbf{A}$. the particle charge and mass number respectively.

Recent work on proton and helium nucleus spectra near solar minimum suggests that solar modulation effects are essentially velocity dependent. 20 The fact that protons and helium nuclei have the same low energy spectrum, in energy per nucleon, indicates that the dominant modulation is a function of velocity alone. For the present analysis, the change-over from the purely velocity dependent function (5) for low energies to the formula (6) for high energies is effected at a kinetic energy per nucleon $\left(E_{0}\right)$ given by

$$
E_{0}=M c^{2}\left\{\left[1+\left(\frac{\ell Z}{R_{0} A}\right)^{2}\right]^{1 / 2}-1\right\}
$$

Figure 3 shows the demodulated spectrum (curve B) thus obtained from the observed spectrum (curve A) for $E_{0}=1 \mathrm{BeV}$. Below approximately $500 \mathrm{MeV}$ per nucleon, curve $B$ exhibits the characteristic of a flat spectrum. We have found that curve $B$ remains essentially the same for all $\left(\ell Z / R_{0} A\right)>1$. Therefore we may fit the data for helium nuclei $(A=2 Z)$ as well as the data for protons $(A=Z)$ by requiring that $\ell / R_{0}>2$. This may be achieved with reasonable values for $\ell$ and $B$ (e.g. $\ell \sim 10^{12} \mathrm{~cm} ., B \sim 10^{-5}$ gauss).

At intermediate energies of a few $\mathrm{BeV}$ the solar modulation could be a sensitive function of the detailed structure of the interplanetary magnetic field. In this paper, we rely primarily upon the purely velocity dependent solar modulation formula (5) for low energies and utilize the indicated hybrid formula (6) merely as a matching function that meets the asymptotic constraint of zero modulation at very high energies. 


\section{EXTRAPOLATION TO THE SOURCE}

By a detailed consideration of ionization losses, the solar demodulated spectrum is extrapolated to the source. Since these particles diffuse through the interstellar medium, the amount of matter traversed exhibits a dispersion about the mean value of $2.5 \mathrm{gm} / \mathrm{cm}^{2}$, and as these energies are low, the inferred source energy of each observed particle depends critically on the actual path traversed. An expression for the distribution of path lengths due to diffusion is derived in the appendix. The square root of the variance of the path lengths associated with this distribution is $(2 / 5)^{1 / 2}$ of the mean path length, and therefore this complication to the extrapolation clearly cannot be ignored.

Each observed particle is, in principle, mapped into a unit modulus energy distribution at the source according to the a priori distribution in path lengths arising from a diffusion process. These individual distributions at the source are weighted by the solar demodulation function, (5) and (6), and the resulting source spectrum (curve C) is shown in Figure 3. This source spectrum exhibits a peak at about $100 \mathrm{MeV}$ per nucleon and falls rapidly at lower energies.

\section{DISCUSSION}

When compared with the observed spectrum, the source spectrum shows the systematic effects of energy loss by ionization, particularly at the low energy end. The smoothness at the low energy end is due to the dispersion in path lengths in the interstellar medium. If all particles were assumed to traverse exactly $2.5 \mathrm{gm} / \mathrm{cm}^{2}$, then the source spectrum thereby inferred would exhibit an abrupt cutoff at about $80 \mathrm{MeV}$ per nucleon. 
Under the assumption that high energy particles (> several BeV) can . freely propagate away from the source accelerator and are not modulated drastically by galactic or solar magnetic fields, the energy spectrum of accelerated particles, at high energies, is the same as the asymptotic form (2) of the observed spectrum;

$$
\frac{d J}{d E}=10^{8} E^{-2.5}
$$

This is shown as curve $D$ in Figure 3.

This spectrum (8) for accelerated particles probably does not remain valid down to extremely low energies, but we know that it is an adequate description for several decades in the region of several BeV. As pointed out by Syrovatskii ${ }^{21}$ this form (8) of the spectrum corresponds to a general situation of energy equipartition among cosmic rays, magnetic fields, and turbulent motion. If we assume that the same form of this spectrum is valid down to about $100 \mathrm{MeV}$, then the ratio of the source intensity (curve C) to the intensity given by curve $D$ is a measure of the transmission efficency of the source envircnment for the energy per nucleon under consideration. Figure 4 shows this transmission coefficient as a function of the reciprocal velocity. The fact that this coefficient exhibits a practically straight line in a semi-log plot with respect to $1 / \beta$ suggests that the accelerated source particles remain partially confined by a process of diffusion. The cosmic ray density "outside the source" is thereby related to the density "inside the source" by a simple relation of the form 


$$
[\mu(E)]_{\text {out }} \propto[\mu(E)]_{\text {in }} \exp \left(-K^{\prime} / \beta\right)
$$

where $K^{\prime}=4.3$, as calculated from the slope of the dashed straight line shown in the transmission ratio plot (Figure 4).

Since the cosmic ray source spectrum appears to be a universal function of velocity rather than rigidity, we infer that the confinement to the source environment does not arise from trapping by ordered magnetic fields. Rather, a comparison of (9) with (5) indicates that the process involved here is one of diffusion, similar to that prevailing in the solar system. This suggests that the magnetic environment of the physical source of cosmic rays might be similar in form to the solar magnetic environment (e.g. magnetic turbulence convected outward by a plasma wind).

\section{ACKNOW LEDGEMENTS}

It is a pleasure to acknowledge the encouragement of Frank B. McDonald and the mathematical counsel of Philip B. Abraham. 


\section{APPENDIX}

The dispersion in cosmic ray particle paths from the source to the solar system is here attributed to a diffusion mechanism. For a particle produced at the space-time origin of a homogeneous isotropic diffusive medium, the probability per unit time of first passage beyond a radius (a) is given by 14

$$
P(t)=\sum_{n=1}^{\infty} 2\left(\frac{n \pi}{a}\right)^{2} D \exp \left\{i(n+1) \pi-\left(\frac{n \pi}{a}\right)^{2} D t\right\}
$$

where $D$ is the diffusion constant characteristic of the medium.

The mean time of first passage $\langle t\rangle$ is constructed as

$$
\langle t\rangle \equiv \int_{0}^{\infty} t P(t) d t=a^{2} /(6 D)
$$

The second temporal moment $\left\langle t^{2}\right\rangle$ is constructed as

$$
\left\langle t^{2}\right\rangle \equiv \int_{0}^{\infty} t^{2} P(t) d t=(7 / 180)\left(a^{2} / D\right)^{2}
$$

The variance $\left(\sigma_{t}\right)^{2}$ of the first passage time is then given by

$$
\left(\sigma_{t}\right)^{2} \equiv\left\langle(t-\langle t\rangle)^{2}\right\rangle=\left\langle t^{2}\right\rangle-(\langle t\rangle)^{2}=\frac{1}{90}\left(a^{2} / D\right)^{2}
$$


By comparing the mean first passage time $\langle t\rangle$, as given by (A2), with the variance, as given by (A4), we note the relation

$$
\sigma_{t}=(2 / 5)^{1 / 2}\langle t\rangle
$$

If we assume that the quantity of matter traversed (S) by an observed particle is proportional to its total transit time, source to observer, then we can use the relation given by (A5) to infer that

$$
\sigma_{s} \equiv\left(\left\langle(S-\langle S\rangle)^{2}\right\rangle\right)^{1 / 2}=(2 / 5)^{1 / 2}\langle S\rangle
$$

We consider a sample of observed cosmic ray particles which is characterized by a certain value for $\langle\mathrm{S}\rangle\left(\mathrm{e} . \mathrm{g} \cdot 2.5 \mathrm{gm} / \mathrm{cm}^{2}\right)$ and assign the corresponding variance prescribed by diffusion theory. The well defined mean $\langle S\rangle$ and square root of the variance $\sigma_{S}(A 6)$ here ascribed to (S) are sufficient to construct a normal Gaussian distribution function:

$$
P(S)=\frac{(2 \pi)^{-1 / 2}}{\sigma_{S}} \exp \left\{-\frac{(S-\langle S\rangle)^{2}}{2\left(\sigma_{S}\right)^{2}}\right\}
$$

Insertion of the explicit expression (A6) of the square root of the variance into (A7) yields

$$
P(S)=\frac{(5 / \pi)^{1 / 2}}{2\langle S\rangle} \exp \left\{-\frac{5}{4}\left(\frac{S-\langle S\rangle}{\langle S\rangle}\right)^{2}\right\}
$$


This distribution function (A8) is renormalized to account for the exclusion of negative values for (S), as follows:

$$
\mathbf{P}^{\prime} \equiv \mathbf{P} /\left(\int_{0}^{\infty} \mathbf{P d S}\right)
$$

where

$$
\int_{0}^{\infty} \operatorname{Pds}=0.9429
$$

This normalization procedure (A9) is based upon the approximation that $\langle S\rangle$ is much greater than the shortest possible path length from the source to the observer. This assumption is valid in practice since the material diameter of the entire galaxy is an order of magnitude less than $\langle S\rangle$.

The final expression of the renormalized distribution function is:

$$
P^{\prime}(S)=\frac{(5 / \pi)^{1 / 2}}{1.8858\langle S\rangle} \exp \left\{-\frac{5}{4}\left(\frac{S-\langle S\rangle}{\langle S\rangle}\right)^{2}\right\}
$$

where

$$
\int_{0}^{\infty} P^{\prime} d s=1.0000
$$


1. M. F. Kaplon and G. Skadron, Nuovo Cimento 34, 1687 (1964).

2. C. J. Waddington, Proc. Kyoto Conf. Cosmic Rays (1961).

3. C. E. Fichtel, Nuovo Cimento 19, 1100 (1961).

4. F. B. McDonald and W. R. Webber, J. Geophys.Res. 67, 2119 (1962).

5. F. W. O'Dell, M. M. Shapiro and B. Stiller, Proc. Kyoto Conf. Cosmic Rays (1961).

6. G. D. Badhwar, R. R. Daniel and B. Vijayalakshmi, Progr. of Theor. Phys. (Kyoto) 28, 607 (1962).

7. Another method for measuring the interstellar matter traversed, similar in principle to that described in the text, is to use the $\mathrm{He}^{3} / \mathrm{He}^{4}$ ratio in cosmic radiation (M. V. K. Apparao and M. F. Kaplon, Proc. of Kyoto Conf. Cosmic Rays, 1961). Though there are some observations on this ratio, there is as yet a paucity of data, particularly for the high energy region, and the method is yet to give information of comparative utility.

8. H. Aizu, Y. Fujimoto, S. Hasegawa, M. Koshiba, Y. Mito, J. Nishimura, K. Yoki and M. Schein, Phys. Rev. 121, 1206 (1961).

9. G. D. Badhwar, S. N. Devanathan and M. F. Kaplon, J. Geophys. Res. 70, $1005(1965)$

10. F. B. McDonald and G. H. Ludwig, Phys. Rev. Letters, 13, 783 (1964). 
11. C. Y. Fan, G. Gloeckler and J. A. Simpson, University of Chicago EFINS$65-22(1965)$

12. V. L. Ginzburg and S. I. Syrovatskii, The Origin of Cosmic Rays (The MacMillan Company, New York, 1964).

13. S. Miyake, J. Phys. Soc. Japan 18, 1226 (1963).

14. A. W. Wolfendale, Proc. Jaipur Conf. Cosmic Rays (1963).

15. P. H. Fowler and C. J. Waddington, Phil. Mag. 1, 637 (1956).

16. E. Lohrmann and M. W. Teucher, Phys. Rev. $\underline{115}, 638$ (1959).

17. C. J. Waddington, Prog. Nuclear Phys. $\underline{8}, 1$ (1960).

18. E. N. Parker, Interplanetary Dynamical Processes (Interscience Publishers, New York, 1963).

19. G. Klein, Proc. Roy. Soc. (London) 211-A, 431 (1952).

20. W. R. Webber, University of Minnesota, Technical Report CR-76 (1964).

21. S. I. Syrovatskii, Soviet Phys. JETP 쓰, 1788 (1961).

22. V. K. Balasubrahmanyan and F. B. McDonald, J. Geophys. Res. 69, 3289 (1964).

23. C. E. Fichtel, D. E. Guss, D.A. Kniffen and K. A. Neelakantan, J. Geophys. Res. 69, 3293 (1964). 


\section{FIGURE CAPTIONS}

Fig. 1 Proton and He nucleus rigidity spectra. The He nucleus data have been multiplied by 7 . The low rigidity data were obtained from the IMP-I satellite during the period November 1963-May 1964 (references 10 , 11). The other data were obtained from balloon measurements during June 1963 (references 22, 23). The dotted curve was calculated by McDonald and Ludwig (reference 10).

Fig. 2 The proton and He nucleus data (reference 10,11, 22, 23) plotted in a kinetic energy per nucleon representation. The He nucleus data have been multiplied by 5.7. The solid line is equation (1).

Fig. 3 The energy per nucleon spectrum as observed (A), solar demodulated (B), corrected for ionization losses in interstellar matter (C), and extrapolated from higher energies, for comparison (D).

Fig. 4 Source transmission coefficient plotted as a function of $1 / \beta$ (solid curve). The slope of the dashed straight line is -1.9 . 


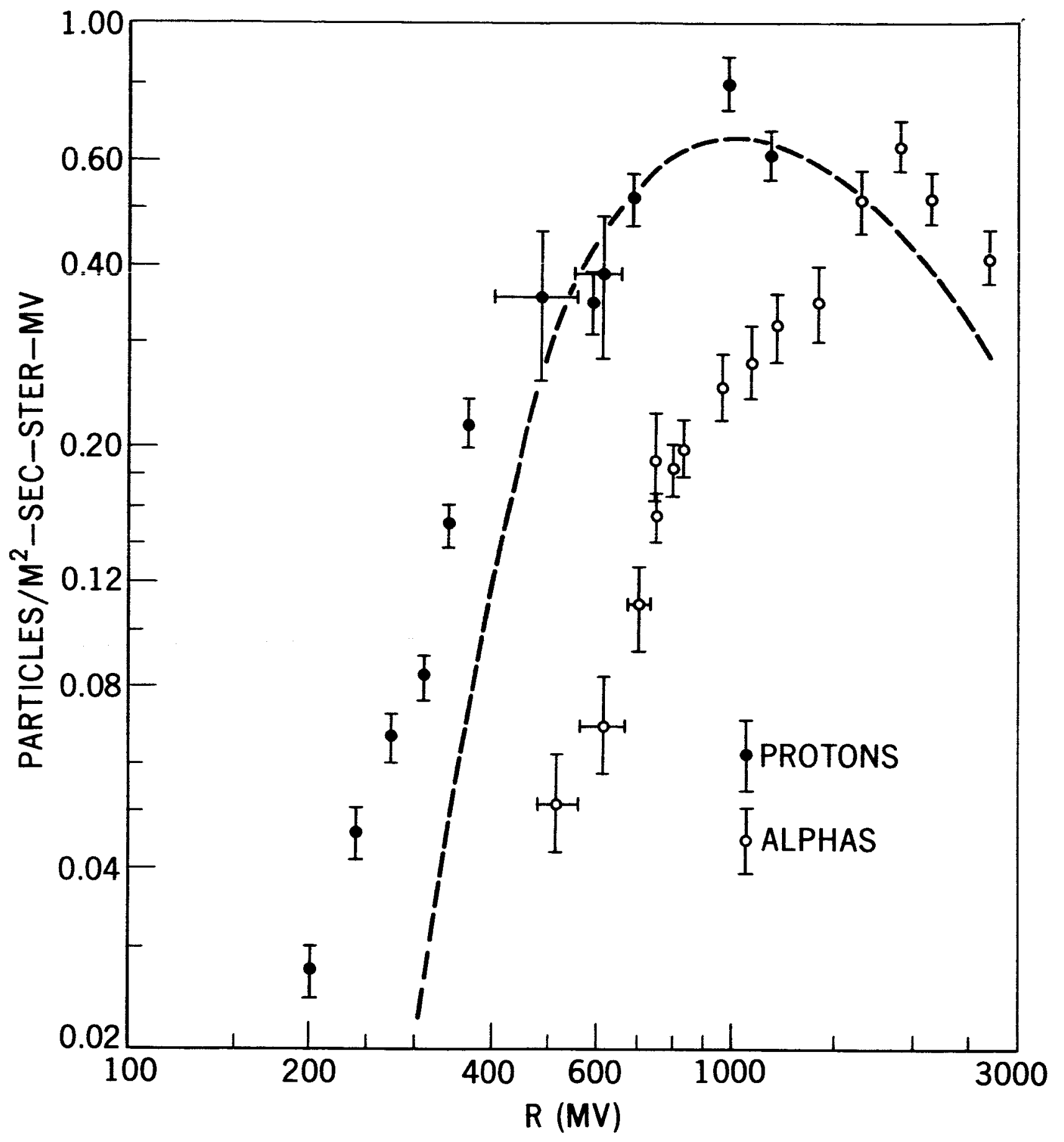




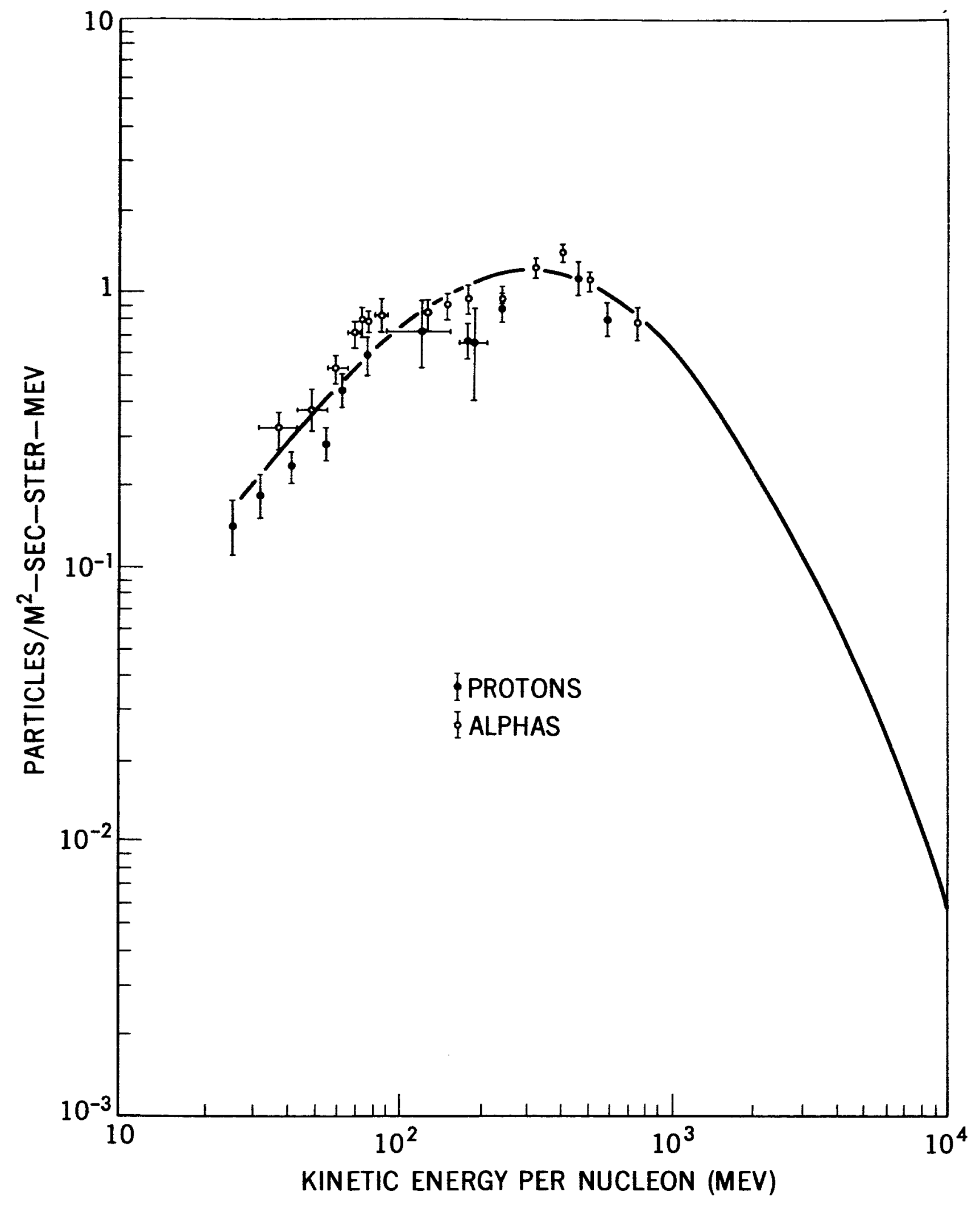

FIGURE 2 


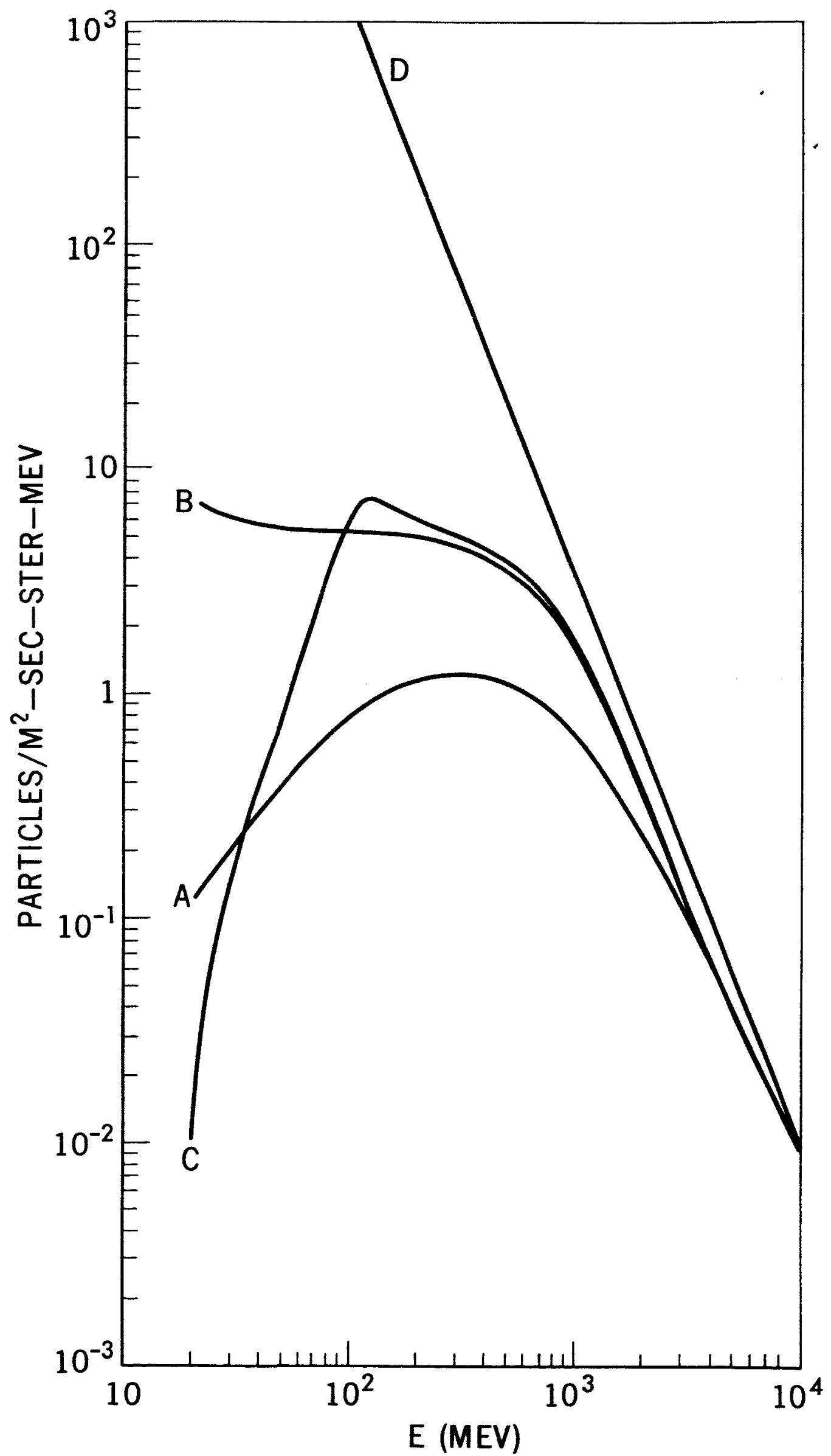

FIGURE 3 


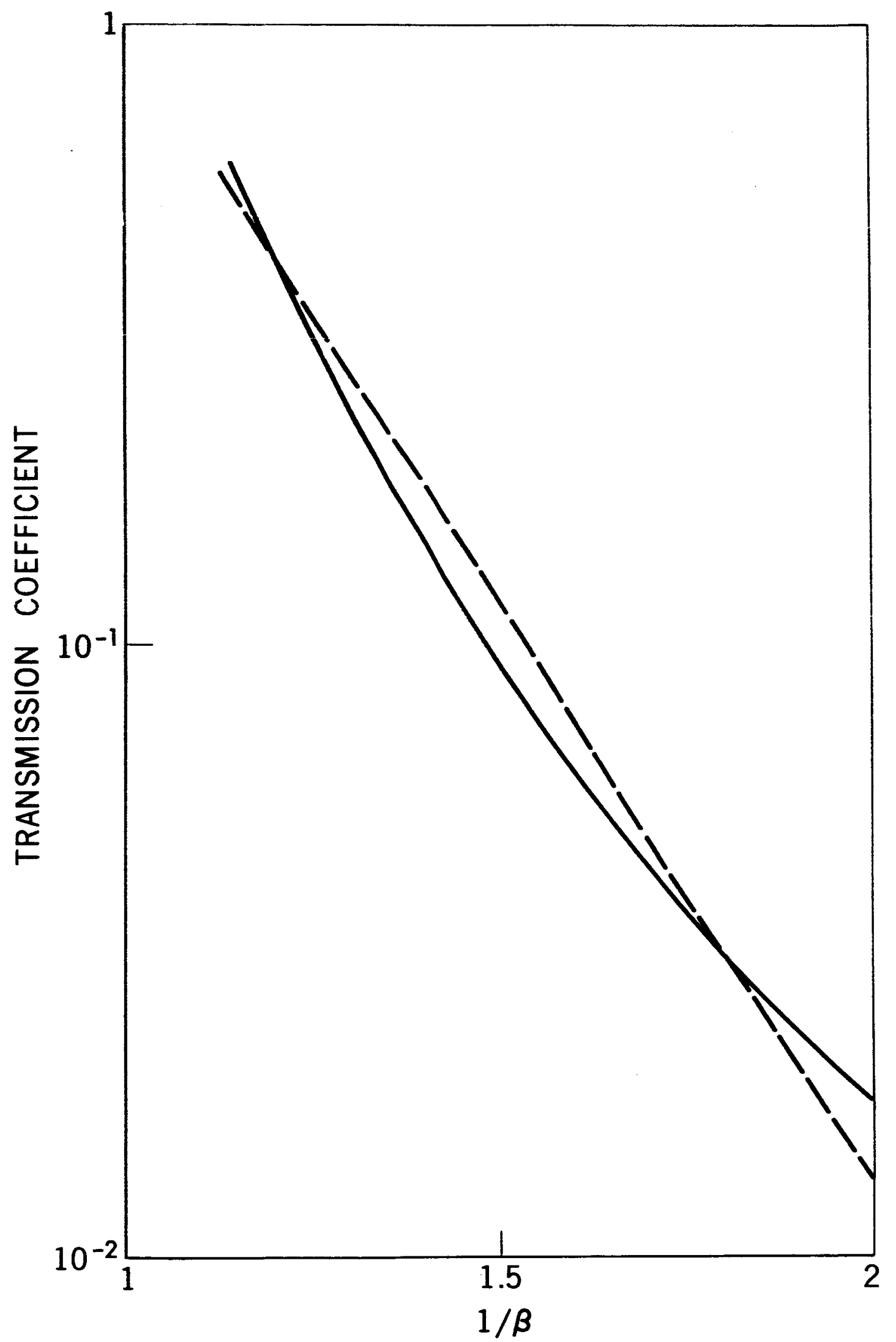

FIGURE 4 